\title{
On the subject of serial killer. Psychological assessment from the handwriting
}

\section{In tema di serial killer. Disamina psicologica dalla scrittura \\ Sobre el asunto del asesino en serie. Evaluación psicológica de la escritura a mano}

\author{
Monica Calderaro \\ Grafologa forense, Criminologa, Dottore in Psicologia ad indirizzo clinico, Responsabile Didattico e Docente del Corso \\ di Formazione in Grafologia Forense, Sapienza Università di Roma, Italy
}

\begin{abstract}
From a research carried out since 2010, at the Chair of Forensic Psychopathology directed by prof. Vincenzo M. Mastronardi of the Sapienza University of Rome and within the teaching of Psychographology at the Masters in Criminology and in more scientific areas, has turned the focus on the research on the psychographic examination of Serial Killer (subject of this article), Sex Offenders, Stalker, Classic Mass Murder, Family Mass Murder, Figlicide Mothers and other criminal phenomena with the intention of highlighting, where existing, graphological characteristics and indices, assimilable to the various subjects responsible for the same type of crime and, therefore, possible 'graphological patterns' to be highlighted as symptomatic graphic imprints of a determinate 'behavioural model'. Proceeding with the graphological comparison, although between different personality structures, certain peculiarities were found, such as, for example, signs which lead back to aggressiveness, a typical trait among others, of the Serial Murderers, including certain deficits in the affectivesexual area. Interesting what emerged from the above mentioned study. Considering that Psychographology is an effective indicator of the structure of the personality, representing, therefore, an excellent revealing instrument of the deepest dynamics of the individual, both at an intra-psychic and interpersonal level (free from any diagnostic hypothesis), writing can be considered, as indicated above, a graphic imprint of the "individual psychism" and the percentage of certainty is about $80 \%$, where, however, there is no possibility of simulating or dissimulating, due to a series of specific factors. The research in the Serial Killer area has already oriented towards the tangible manifestation of aggressive instances, significant problems in the affective-sexual sphere, as well as to a particularly accentuated narcissistic trait. The serial murders are of a personal nature and the application of the behavioural analysis through the psychographic reading key, orients us to understand the deeper dynamics that have been triggered off in the subject, thus answering also some famous questions of the criminology such as, for example, why and what drove him to commit such crimes.
\end{abstract}

\section{RIASSUNTO}

Da una ricerca condotta già dal 2010, presso la Cattedra di Psicopatologia forense diretta dal prof. Vincenzo M. Mastronardi della Sapienza Università di Roma e nell'ambito dell'insegnamento di Psicografologia presso i Master in Criminologia ed in più ambiti scientifici, si è rivolto il focus attentivo, sulle ricerche relative alla disamina psicografologica in tema di Serial Killer (oggetto del presente articolo), Sex Offenders, Stalker, Classic Mass Murder, Family Mass Murder, Madri Figlicide ed altre fenomenologie criminali con l'intento di evidenziare ove esistenti, caratteristiche ed indici grafologici, assimilabili ai vari soggetti responsabili dello stesso tipo di reato e quindi possibili 'pattern grafologici' da evidenziare a mò di impronta grafica sintomatica di un determinato 'modello comportamentale'. Procedendo alla comparazione grafologica, sia pur tra differenti strutture di personalità, si sono riscontrate alcune peculiarità come, ad esempio, i segni che riconducono all'aggressività, tipico tratto distintivo tra gli altri, degli Assassini Seriali, ivi inclusi determinati deficit nell'area affettivo-sessuale. Interessante quanto emerso dal suindicato studio. Considerando che la Psicografologia è un indicatore efficace della struttura di personalità rappresentando quindi un ottimo strumento rivelatore delle dinamiche più profonde dell'individuo sia a livello intrapsichico che interpersonale, (scevro da qualsivoglia ipotesi diagnostica), la scrittura può essere considerata come suindicato, una impronta grafica dello "psichismo individuale" e la percentuale di certezza è dell' $80 \%$ circa, li dove peraltro non vi è possibilità di simulare o dissimulare, per una serie di fattori specifici. La ricerca nell'ambito dei Serial Killer ha già orientato verso la tangibile estrinsecazione di istanze aggressive, significative problematiche nella sfera affettivo-sessuale, nonché ad un tratto narcisistico particolarmente accentuato. Gli omicidi seriali sono di natura personale e l'applicazione dell'analisi comportamentale mediante la chiave di lettura psicografologica, ci orienta a comprendere le dinamiche più profonde che si sono innescate nel soggetto, rispondendo quindi anche ad alcuni famosi interrogativi della criminologia quali per esempio, il perché e il cosa lo ha spinto a commettere tali reati.

\section{RESUMEN}

De la investigación realizada desde 2010, en la Cátedra de Psicopatología Forense dirigida por el prof. Vincenzo Mastronardi de la Universidad Sapienza de Roma y en el contexto de la enseñanza de Psicografía en el Máster en Criminología y en campos más científicos, se enfocó la atención, en la investigación relacionada con el examen psicografológico sobre el tema de Serial Killer (tema de este artículo), Delincuentes sexuales, Acosador, Asesinato en masa clásico, Asesinato en masa familiar, Madres Filicidas y otras fenomenologías criminales con la intención de resaltar dónde existen, características e índices grafológicos, similares a los diversos sujetos responsables del mismo tipo de delito y por lo tanto, es posible que se resalten 'patrones grafológicos' como una impresión gráfica sintomática de cierto 'modelo de comportamiento'. Continuando con la comparación grafológica, aunque entre diferentes estructuras de personalidad, se encontraron algunas peculiaridades como, por ejemplo, los signos que conducen a la agresividad, un rasgo distintivo típico entre otros, de los Asesinos en Serie, incluidos ciertos déficits en el área afectiva. sexual. Interesante lo que surgió del estudio antes mencionado. Teniendo en cuenta que la psicografía es un indicador efectivo de la estructura de la personalidad, por lo tanto, representa una excelente herramienta 
para revelar la dinámica más profunda del individuo tanto a nivel intrapsíquico como interpersonal (sin ninguna hipótesis diagnóstica), la escritura puede considerarse como se indicó anteriormente, una impresión gráficos del "psiquismo individual" y el porcentaje de certeza es de aproximadamente el $80 \%$, donde, sin embargo, no existe la posibilidad de simular o disimular, para una serie de factores específicos. La investigación en el campo de los asesinos en serie ya se ha orientado hacia la manifestación tangible de casos agresivos, problemas significativos en la esfera afectivo-sexual, así como un rasgo narcisista particularmente acentuado. Los asesinatos en serie son de naturaleza personal y la aplicación del análisis del comportamiento a través de la clave de lectura psicografológica nos dirige a comprender la dinámica más profunda que se desencadenó en el tema, respondiendo así algunas preguntas famosas de criminología como, por ejemplo, por qué y qué condujo él para cometer tales crímenes.

\section{La psicografologia}

Considerando che la scrittura è come un'impronta digitale (una sorta di DNA grafico), in quanto il soggetto nel gesto grafico proietta la parte inconscia quindi lo "psichismo individuale", è importante in questo caso sostenere, che "Non è il singolo segno a determinare questa o quella tipologia di caratteristiche psicologiche, bensi il concorso di diversi segni che talvolta tendono a bilanciarsi neutralizzandosi tra loro o talvolta a rinforzarsi vicendevolmente".

Viepiù, nella disamina di un profilo psicografologico (scevro da qualsivoglia diagnosi) e rivolto all'evidenziazione dei "Tratti della personalità", è della massima importanza considerare il contesto grafologico che si esamina, in quanto molto spesso, la chiave di lettura (a differenza del Falso documentale mirato all'evidenziazione di più segni cosiddetti "coattivi", tipici dello "psichismo individuale", che lì dove ripetuti più volte, ci consentono di affermare se o meno un documento come per es. un testamento, appartiene alla persona che lo ha vergato), nel test psicografologico, va sempre osservato tutto il contesto della scrittura, dove alcuni segni possono assumere in un determinato quadro grafologico, un significato diverso dalla caratteristica generale, vedi ad esempio un tratto grafico con direzione del rigo ascendente indice generalmente di un tratto della personalità idealista e che aspira al raggiungimento dei propri obiettivi, in un tracciato molle e con scarsa energia, rivela invece, una lotta ad un "fundus depressivo".

Considerato quanto su scritto ed in relazione quindi al supporto che il test grafologico fruisce alla già disamina onnicomprensiva (vedi ad esempio nell'ambito criminologico-forense), mediante il colloquio clinico e quando ritenuto necessario, con l'ausilio di test psicodiagnostici (cosiddetti universalmente accettati), attualmente la comunità scientifica, si sta sempre più sensibilizzando all'utilizzo anche del test grafologico che indubbiamente, si rivela di utile supporto in grado di cogliere alcuni elementi e caratteristiche essenziali al completamento di un profilo personologico (vedi ad es. tra gli altri, il recente utilizzo di questo test nel caso di Cogne in persona di Annamaria Franzoni, condotto dal prof. Alberto Bravo).

Viepiù, nei casi in cui il soggetto è scomparso o deceduto, si rivela estremamente importante il reperimento di saggi grafici in grado di fornici (in questo caso), l'unico strumento possibile per poter risalire al 'modus vivendi ed operandi' del soggetto e quindi il proprio modo di comportarsi, il tipo di relazioni sociali e soprattutto con il rapporto con il proprio "Sé", che potrà essere in grado di fornirci attraverso la disamina di determinate caratteristiche grafologiche, il 'Profilo della Personalità'.

Prima di affrontare l'ambito squisitamente criminologico che vedrà la disamina grafologica di tre assassini seriali, in estrema sintesi e per la curiosità del lettore, si riportano qui di seguito le Competenze specifiche dell'esperto grafologo:

- Perito esperto in perizie grafiche (settore giudiziario) autenticità di documenti aventi valore legale, ovvero falsi grafici su (testamenti, atti pubblici, assegni, ecc.);

- Consulenza aziendale - Si applica sulla selezione ed assunzione del personale, ossia la disamina mirata alle congenialità del singolo candidato per un incarico specifico;

- Orientamento scolastico - Come sostegno nei bambini e negli adolescenti durante il percorso scolastico, mirato alle scelte esistenziali, ai fini di una congeniale scelta professionale);

- Profilo psicografologico - Sia in ambito criminologicoforense che terapeutico individuale, familiare e/o per le dinamiche di coppia.

\section{Fenomenologia dell'omicidio seriale}

Prima di procedere alla Disamina relativa al profilo grafologico di un omicida seriale, è doverosa una sia pur sintetica premessa sul tema e le relative dinamiche che vi sono a monte, quali ad esempio le motivazioni, movente, ecc. Molteplici sono le teorie e le definizioni attribuite ai Serial Killer, tuttavia, la maggior parte concordano sull'assunto che trattasi di individui che commettono tali reati per motivazioni prevalentemente basate su elementi quali predominio, sesso e vissuto di onnipotenza dato da un tratto narcisistico particolarmente elevato e che per essere considerati assassini seriali, devono commettere una serie di omicidi (da 2-3 in poi) in un determinato arco temporale, seguendo rituali specifici che vanno a costituire quella che viene definita "La Firma". Vi sono poi Serial Killer che deviano dalla suindicata descrizione e per questo ritenuti anche 'atipici'. L'FBI 1979 (Federal Bureau of Investigation), divide gli assassini multipli in tre sottocategorie:

- Serial Killer "assassino seriale" che secondo Douglas e Olshaker (1999), uccide da tre a più vittime, in luoghi diversi e tra un omicidio e l'altro, si verifica il "cooling-off" (intervallo emotivo) di cui la durata può variare da settimane, mesi e/o anni. Le vittime possono essere scelte a caso o in base a determinate caratteristiche. Ciò che lo distingue, è l'azione delittuosa ripetuta con motivazioni distinte rispetto ad altre tipologie di omicidi.

- Mass Murder "assassini di massa" che colpiscono in un luogo preciso uccidendo diverse vittime e in un unico evento. Generalmente i luoghi scelti sono molto affollati come ad es.: MacDonald, Scuole, ecc. La causa scatenante può essere una forte ferita narcisistica come ad esempio un abbandono, licenziamento ecc.

- Spree Killer in stato di coscienza alterata, "assassino compulsivo" che uccide in un lasso di tempo decisamente breve da due o più vittime in una sorta di "raptus omicida" ed in luoghi diversi in seguito ad un evento scatenante e dove l'omicidio diviene una sorta di 'baldoria' in stile "Arancia Meccanica". 


\section{Il profilo psicologico del serial killer}

Holmes e DeBurger (1990), hanno concentrato le loro ricerche prevalentemente sui serial killer e i delitti a carattere di mostruosità in genere. Tali Autori ritengono che il contesto socioculturale sia dell'assassino che della vittima, può servire da cofattore nella genesi dell'omicidio seriale riferendosi specificamente a problematiche sociali (negli Stati Uniti) quali anomia, deumanizzazione da parte della società, eccesso di violenza, mobilità ecc.

Grazie alla disamina rilevata dai dati su 110 omicidi seriali, la Sezione di Scienza del Comportamento dell'FBI (1990), concentrò l'attenzione sulla personalità e miratamente alle motivazioni che spingono a compiere l'atto omicida, espletando una Classificazione di tipi di Serial Killer (modificata da Palermo e Mastronardi 1995), sviluppando il cosiddetto "Profilo" sulla base di criteri mirati a "modus operandi", "area geografica", "firma" ed in alcuni casi attività di "staging"(voluta alterazione della scena criminis):

- Il tipo Visionario;

- Il tipo Missionario;

- Il tipo Edonista;

- Il tipo orientato al Controllo del Potere;

- Il tipo Lussurioso;

di cui la panoramica onnicomprensiva relativa alle specificità delle suindicate tipologie ed altro ancora, si rimanda al volume: Mastronardi-Palermo "Il Profilo Criminologico" (Op. cit.).

Pertanto l'attività di Profiling rivolta all'espletamento del profilo dell'ipotetico autore del reato, si articola e si costruisce sulla base di un insieme correlati valutativi mirati, ricavati dall'osservazione della scena del crimine e di cui l'assunto di base del Profiling come processo evolutivo, parte dai famosi 6 interrogativi anglosassoni della criminodinamica (Cosa - What?; Come - How?; Quando - When?; Dove - Where?; Perché Why?; Chi - Who?), a vantaggio soprattutto di quel tipo di crimine apparentemente senza un movente con modalità omicidiaria cruenta.

Sempre dal succitato Volume (Mastronardi - Palermo), si riporta qui di seguito, la Tabella relativa alla psicodinamica del Serial Killer del Controllo del Potere, che ben si connota ai 3 soggetti scelti per disamina grafologica.

- Sentimenti di inadeguatezza relazionale;

- Frustrazione e collera per insoddisfazione dei propri bisogni relazionali sessuali;

- Negazione a se stesso delle proprie negatività;

- Fantasie sessuali compensatorie insoddisfatte (masturbazione);

- Distorsione cognitiva: 1. Attribuzione di colpa: "Sono le donne responsabili di avermi provocato questa forma di tensione nervosa con il loro sesso" (cfr. Patrick Byrne, 28 anni - 1959, Serial Killer di origine irlandese); 2. Deumanizzazione della donna ("donna oggetto");

- Elaborazione di fantasie devianti di "possesso ad ogni costo";

- Autoinganno: immediata necessità di liberarsi da eventuali sensi di colpa per mezzo di razionalizzazioni soggettive o diniego circa l'atto compiuto: "E'stata lei a provocarmi";

- Strutturazione di meccanismi di appetizione (addiction) per ragioni di gratificazione soggettiva sia pur perversa di possesso: "Sono stato capace finalmente di fare qualcosa di grande, ho veramente coraggio, sono in gamba".

(Palermo - Mastronardi "Il Profilo Criminologico" pag. 168)
Lo schema su riportato, ben si articola con le fasi di Norris (1988)

- Aurorale (fantastica sulla propria vittima);

- di Puntamento (ricerca della vittima ideale);

- della Seduzione (avvicinamento mediante strategie per conquistarne la fiducia);

- della Cattura (obiettivo raggiunto);

- Omicidiaria (trionfo e potere sulla propria vittima in balia dell'aggressore);

- Totemica (terminata la fase dell'eccitazione, molti assassini seriali, sprofondano in una fase depressiva e per cercare di ritardarla, solitamente si servono dei cosiddetti 'souvenir');

- Depressiva (fase in cui per riuscire a provare il medesimo eccitamento, devono ripetere il suindicato ciclo.

\section{Serial Killer. Profilo psicologico dall'analisi della scrittura.}

Ted Bundy, John Wayne Gacy, Jeffrey Dahmer
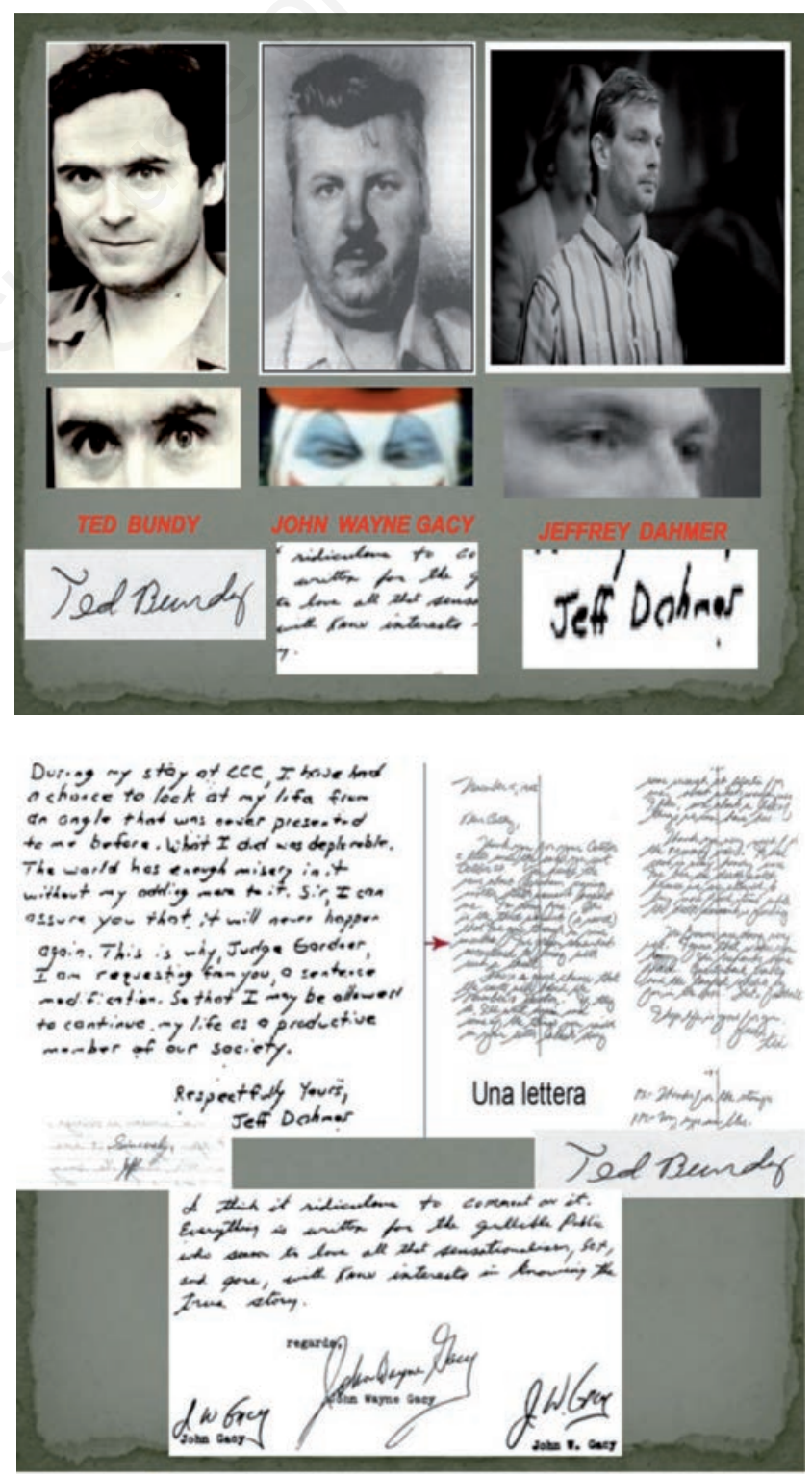
Breve biografia di Jeffrey Dahmer

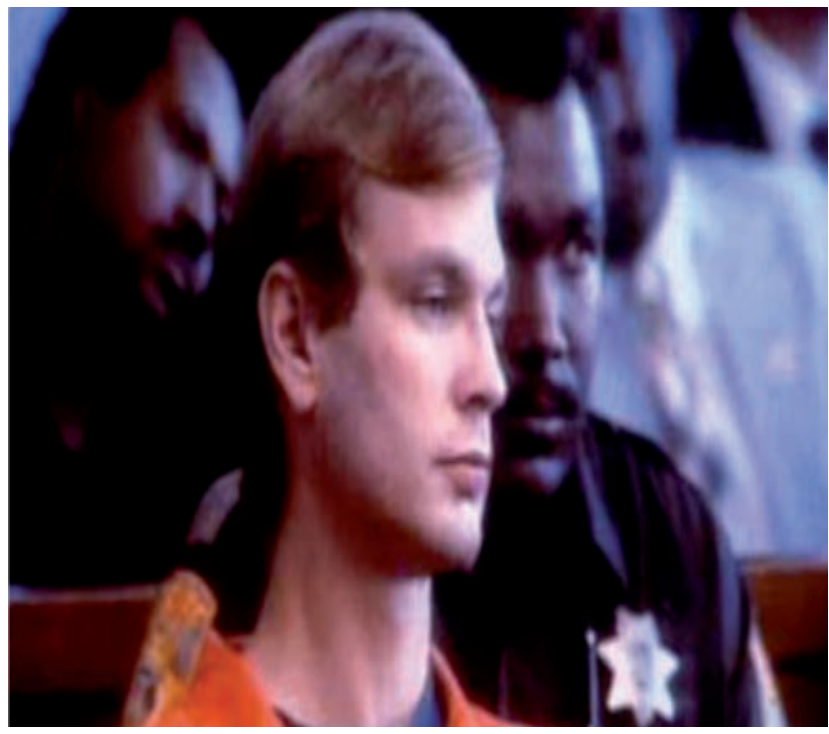

Soprannome: Il Cannibale di Milwaukee

Luogo omicidi: USA, Milwaukee, Wisconsin

Periodo omicidi: 1978-1991

Numero vittime: 17

Modus operandi: Torturava, faceva a pezzi i corpi e ne mangiava alcune parti

Cattura: 22 luglio 1991

Condanna: Ergastolo per ogni omicidio commesso, totalizzando 957 anni di prigione

Epilogo: Ucciso in carcere il 28 novembre 1994 da un detenuto

\section{Le sue parole prima della sentenza}

"Ora è finita. Qui non si è mai trattato di cercare di essere liberato. Non ho voluto mai la libertà. Sinceramente, volevo la pena capitale per me stesso. Qui si è trattato di dire al mondo che ho fatto quello che ho fatto, ma non per ragioni di odio. Non ho odiato nessuno. Sapevo di essere malato, o malvagio o entrambe le cose. Ora credo di essere stato malato. I dottori mi hanno parlato della mia malattia, e ora mi sento in pace. So quanto male ho causato.... Grazie a Dio non potrò più fare del male. Credo che solo il Signore Gesù Cristo possa salvarmi dai miei peccati... Non chiedo attenuanti".

\section{Risultanze perizia psichiatrica: cenni}

George B. Palermo contrastò, in qualità di patologo forense, la difesa che invocava per il cannibale l'infermità mentale e affermò con chiarezza che quel "ragazzone intelligente e garbato, forse solo un po' distaccato e freddo, era perfettamente capace di intendere, di volere, di premeditare e di attuare con lucidità ogni sua singola azione, quindi sano di mente". Una perizia coraggiosa ed esemplare che ancora oggi fa scuola di psichiatria e di giurisprudenza nel mondo e che, allora, costò, a quel mostro non pazzo, la condanna alla massima pena possibile.

\section{Scrittura di Jeffrey Dahmer}

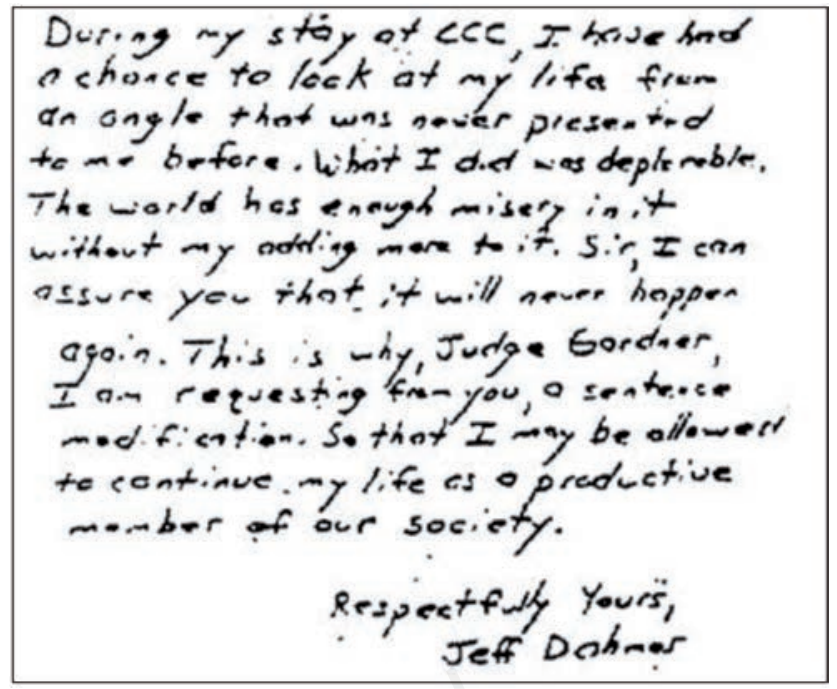

Principali caratteristiche della scrittura, mirate all'evidenziazione di tratti distintivi inerenti le seguenti problematiche: Aggressività, Sessualità, Affettività, Relazioni interpersonali, Scarsa autostima

- Spazio grafico ben riempito con margini irregolari (Necessità di attenzione da parte degli altri, vissuto problematico con le figure genitoriali)

- Tratto irregolare (notevole difetto di logica e di continuità affettiva, soffre di carenze affettive)

- Distanza tra lettere (diminuzione del senso critico)

- Distanza tra parole (necessità di prendere le distanze interpersonali)

- Scrittura slegata (difficoltà di sintesi, discontinuità affettiva)

- Punti sulle i alti (attribuzione massima a particolari minimi)

- Allunghi inferiori pronunciati ed ispessiti (accentuata tendenza alle esperienze di tipo materiale/sessuale a danno dell'obiettività - asola nella lettera "g" incompleta e rifiutata = sfiducia nella congiunzione sessuale"

- Da pendente (inclinata a dx) a eretta (verticale) (vedi particolare nella parola "life") (momentaneo slancio verso l'interpersonale ma pronto a tornare su sé stesso)

- Scrittura tendente al femminile (accentuata sensibilità)

- Virgole grandi e distaccate dal testo (tendenza alla spinta aggressiva)

- Tagli "t" bassi: (tendenza alla rinuncia ai "diritti morali" non mi interessa se gli altri mi giudicano o mi condannano)

- Occhielli scoperti in basso nella lettera "a" e a destra nella lettera "g" (sensibilità verso le cose materiali e sessua$\underline{\text { li giammai spirituali, idealistiche o artistiche) }}$

- Firma distante dal testo con nome staccato dal cognome, quest'ultimo meno comprensibile che si schiaccia nel procedere (presa di distanza tra sé e gli altri. Maggiore importanza a sé stesso rispetto al 'clan di provenienza' (rapporto disfunzionale con la figura maschile [padre]).

\section{Profilo grafologico (sintesi)}

Da quanto emerso dalla disamina grafologica, in Jeffrey Dahmer si evidenziano significative carenze affettivo-relazionali, con necessità di attirare l'attenzione da parte degli altri, probabilmente a causa di un vissuto disfunzionale con le figure genitoriali 
che presumibilmente non hanno soddisfatto le sue istanze affettive, per questo l'omicidio e le modalità messe in atto, rappresentavano l'unica sua forma di espressione.

Il suo quadro grafologico è l'esempio della solitudine, nonostante gli sforzi di approcciarsi con l'altro, non riusciva comunque ad interagirvi con conseguente emarginazione sociale. Tale vissuto di rifiuto e di senso di inadeguatezza lo hanno spinto ad una forma di compensazione., con la necessità di esprimere in senso deviante il possesso dell'altro essere umano.

Caratterizzato da un 'IO' deficitario, non in grado di mediare le richieste impulsive, a tutto vantaggio di un marcato difetto di logica con conseguente diminuzione del senso critico, dove le regole date dalla coscienza morale, erano totalmente svanite.

L'autocontrollo ed il distacco emozionale, spiegano il vigile 'modus operandi', e la capacità di resistere allo stress, essendo in grado di manipolare le vittime, ivi compresa (stando alla descrizione della sua storia biografica), la polizia di tre Stati, facendo di lui un soggetto capace di infondere fiducia negli altri sia pur apparentemente.

Pertanto il conflitto interiore caratterizzato dalla necessità di sentirsi accettato e l'impossibilità di comunicare a causa di 'marcati deficit comunicazionali', associati a 'nuclei ossessivi', lo portavano verso un vissuto di rabbia ed aggressività particolarmente accentuate che evidentemente nel momento dell'actingout, ha commesso gli omicidi come unica forma di soddisfacimento di un'aggressività repressa estrinsecata solo nel momento omicidiario, con conseguente 'malato' piacere sessuale, in grado di farlo sentire narcisisticamente importante solo mediante tale $\underline{\text { evento, }}$ per poi farlo sprofondare nuovamente in quella fase depressiva, di vissuto di solitudine e disagio inaccettabili.

Appartiene alla tipologia del S.K. del 'Controllo del Potere'. Ritenuto capace di intendere e di volere, quindi, 'sano di mente'.

\section{Breve biografia di Ted Bundy}

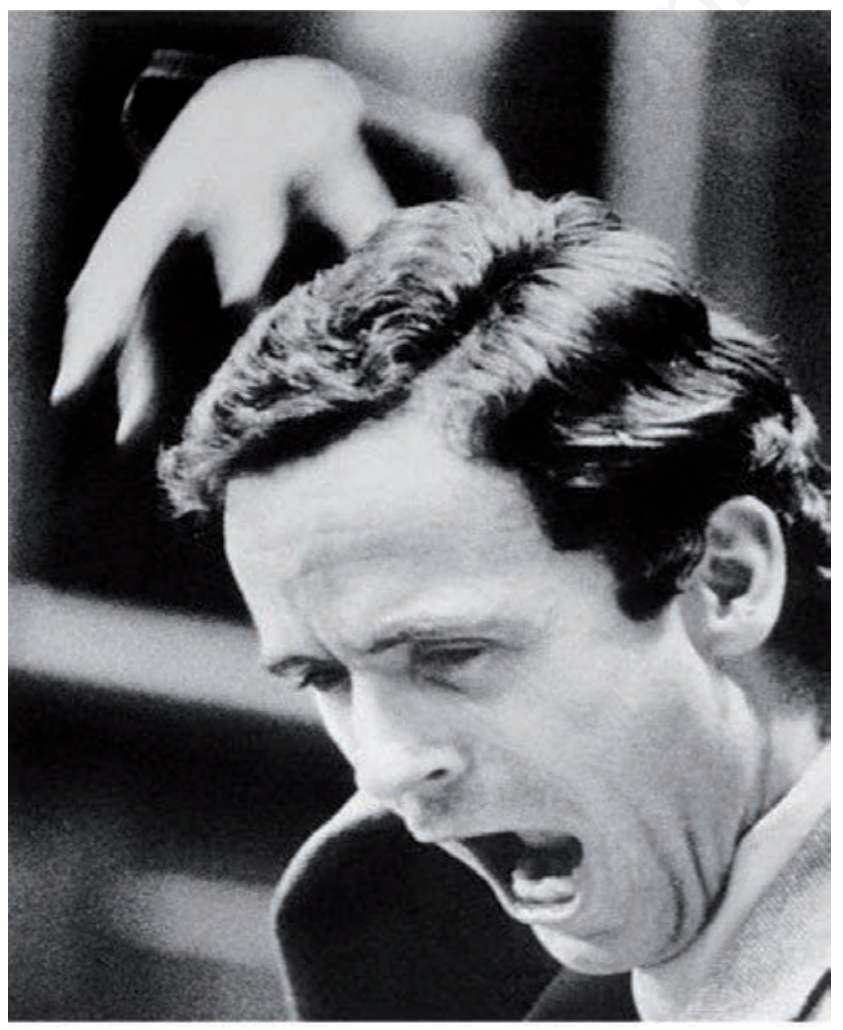

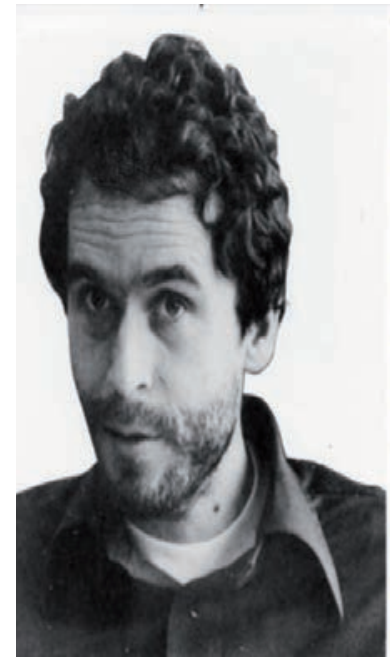

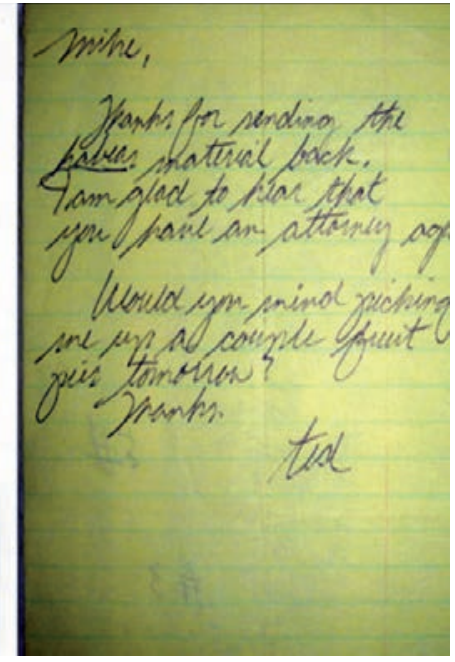

Nome: Theodore Robert Bundy “Ted” Bundy

Soprannome: Lady Killer, Killer delle Studentesse

Nascita: Burlington, 24 novembre 1946

Morte: Florida, 24 gennaio 1989

Vittime accertate: $30-35$

Vittime sospettate: $35-50+$

Periodo omicidi: Presumibilmente dal 13 agosto 1961 o dal 1969; $1^{\circ}$ febbraio $1974-9$ febbraio 1978

Luoghi colpiti: Colorado, Florida, Idaho, Oregon, Utah, Washington, California

Metodi uccisione: Assalto con arma bianca, strangolamento; solitamente attirava a sé la vittima con degli stratagemmi

Altri crimini: Stupro, rapina aggravata, evasione, tentato omicidio, atti di necrofilia e mutilazione

Arresto: 15 febbraio 1978; in precedenza evase due volte

Provvedimenti: Sedia elettrica

Periodo detenzione: 15 febbraio 1978-24 gennaio 1989

\section{Risultanze perizia psichiatrica: cenni}

Fu sottoposto ai tradizionali Test MMPI e TAT (lui aveva studiato psicologia). da Diagnosi fu di "Personalità passivo-aggressiva". Il risultato del suo QI risultò superiore alla media anche se lui cercò di manipolarlo. Su richiesta della difesa, fu disposta una Perizia Psichiatrica, i due Psichiatri che lo esaminarono, lo ritennero capace di intendere e di volere pur se evidenziava un comportamento antisociale. Venne giudicato 'sano di mente'. $\mathrm{La}$ psichiatra Dorothy Otnow sostenne che l'imputato era affetto da sindrome 'maniaco-depressiva', presentando un interessante relazione sulla storia biografica di Bundy inerente a un conflittuale rapporto con suo nonno e la figura materna che nei primi mesi di vita lo lasciò in un Istituto, l'Elizabeth Lund Home for Unwed Mothers. 


\section{Scrittura di Ted Bundy}

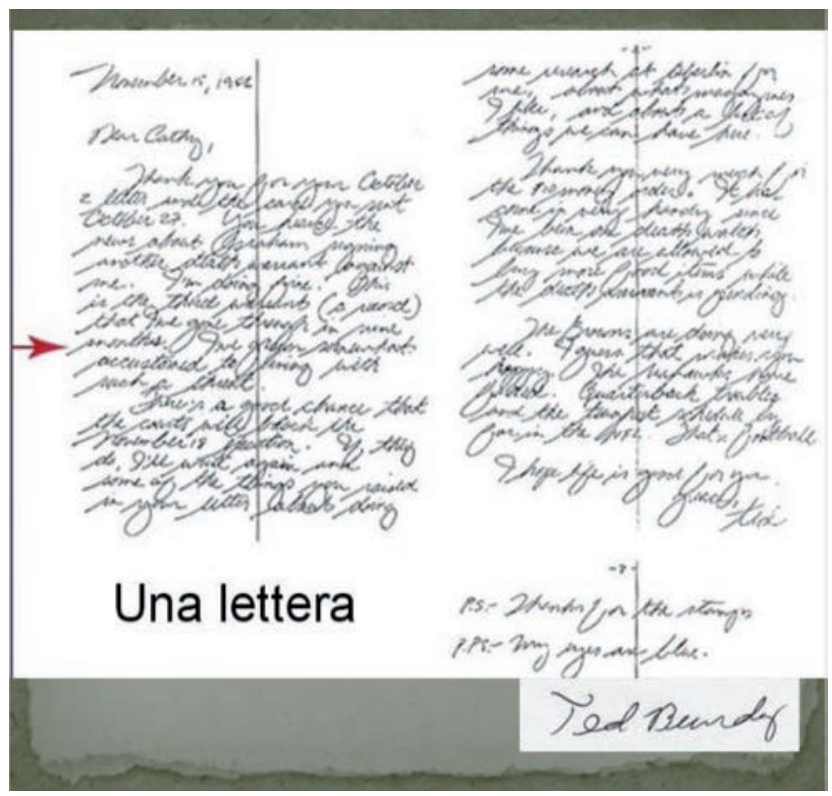

Principali caratteristiche della scrittura, mirate all'evidenziazione di tratti distintivi inerenti alle seguenti problematiche: Narcisismo, Aggressività, Impulsività, Sessualità, Affettività, Relazioni interpersonali

- Spazio grafico riempito (al centro del foglio) (desiderio di sentirsi al centro dell'attenzione. Narcisismo);

- Margini irregolari - molto spaziata a sinistra, destra progressiva (distacco dal passato con eccessiva volontà di proiettarsi verso il raggiungimento delle proprie mete);

- Oscura (il testo incomprensibile, indica volontà di occultamento);

- Rapida (sbrigatività. Continuità del pensiero);

- Stretta tra parole (tendenza all'imprudenza nei rapporti);

- Intricata (tendenza alla confusione nelle facoltà attentive e decisionali. Volontà di predominio, aggressività. Tipico indice della personalità 'fallica');

- Interrigo stretto (eccessiva confusione tra materialità e idealismo);

- Da legata a molto legata (desiderio di non interrompere i propri pensieri ed azioni);

- Tratto appoggiato (desiderio di farsi notare. Aggressività);

- Pendente 'molto inclinata a destra (imprudenza nei rapporti interpersonali);

- Ricci della fissazione materialistica (notevole tendenza alle scorrettezze etiche e materialistiche);

- Tratto " $t$ " nella firma superuncinato (aggressività verbale tendenzialmente amputata);

- Lettere "g" con asole dilatate (accentuazione della stimolazione organico-sessuale);

- Identità tra testo e firma (il vissuto del proprio 'sé' e l'etichetta sociale esibita, sono rappresentati nello stesso modo).

\section{Profilo grafologico (sintesi)}

La problematica di fondo di Bundy è l'abbandono e la delusione affettiva. Dalle evidenze della sua storia, prima subisce l'abbandono da parte della madre e successivamente della sua fidanzata.

La scrittura particolarmente inclinata (fa immaginare un cavallo al galoppo con ritmo spasmodico e disinibito), indice di eccessiva imprudenza nei rapporti interpersonali con cambiamenti repentini del tono dell'umore che varia tra l'eccitato e il depres$\underline{\text { so (vedi la direzione discendente a fine rigo). E' evidente la compo- }}$ nente etero aggressiva, data da forti e compulsivi lanci della scrittura soprattutto nella zona bassa caratterizzata da allunghi eccessivi e dilatati evidenzianti un'esagerata ed incontrollata spinta materiale-sessuale, dove l'altro diviene solo uno strumento per il raggiungimento della propria meta pulsionale da soddisfare nell'immediatezza, tipico indicatore della prevalenza dell'ES (istanza pulsionale) a svantaggio di un 'IO' schiacciato e sopraffatto.

La scrittura presenta inoltre forti intricamenti (le righe si scontrano fra loro), che denotano confusione nelle capacità attentive $\mathrm{e}$ decisionali, nonché eccessiva prevaricazione in modo aggressivo/compulsivo.

Inoltre, la marcata inclinazione degli assi letterali verso destra, denotano una personalità decisamente abile nella fase della "seduzione" (di Norris), che inevitabilmente ha trovato riscontro in molte vittime. Viepiù, emerge la forte spinta narcisistica di chi vuole sentirsi importante ed al centro dell'attenzione, come difesa e compensazione di chi non si sente ascoltato, scegliendo di imporsi sull'altro in modo arrogante.

A differenza di Dahmer, dove il predominio sull'altro era più rivolto per compensare un vissuto di inadeguatezza tale da renderlo esageratamente timido nello scambio relazionale scegliendo quindi l'unica modalità per lui possibile di condivisione relazionale, in Bundy il movente appare più di tipo rivendicatorio di colui che non riuscendo a sopportare la ferita narcisistica, decide in modo deviato, di punire a sua volta tutte quelle donne che andavano a rappresentare per lui, un vissuto del passato, anche se entrambi colpiti a livello affettivo sia pur per fattori diversi. Appartiene alla categoria del S.K. del 'Controllo del Potere'. Ritenuto capace di intendere e di volere, quindi, 'sano di mente'.

\section{Breve biografia di John Wayne Gacy}

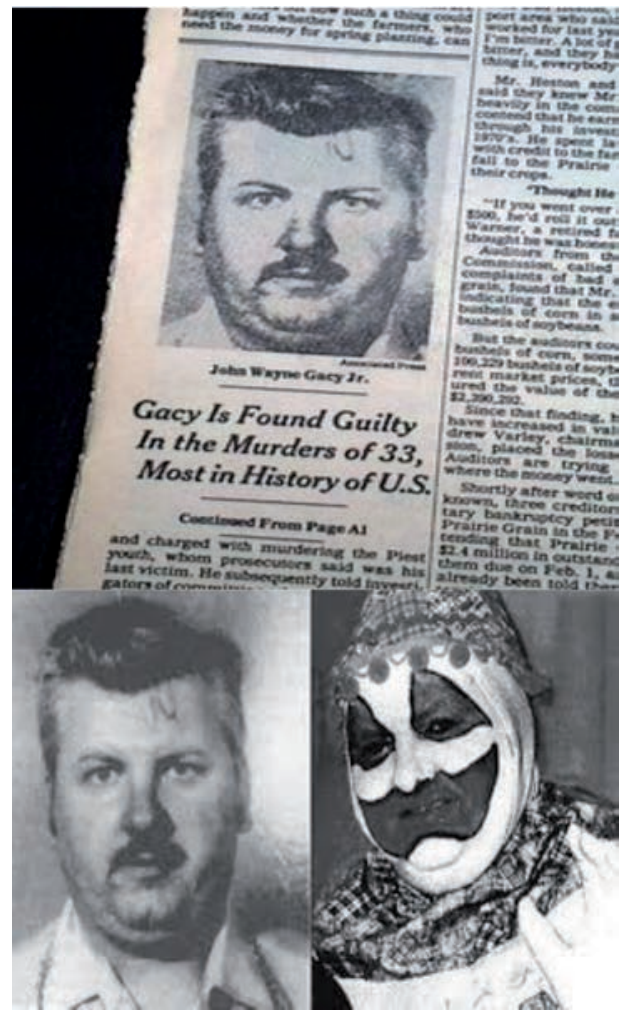


Soprannomi: Pogo the Clown, Killer Clown

Nascita: Chicago 17 marzo 1942

Morte: Crest Hill, 10 maggio 1994

Vittime accertate: 33

Periodo degli omicidi: 3 gennaio 1972 - 11 dicembre 1978

Luoghi colpiti: Des Plaines

Metodi di uccisione: Strangolamento, armi da taglio

Altri crimini: Sodomia, stupro, tortura, rapimento

Arresto: 22 dicembre 1978

Provvedimenti: Condanna a morte tramite iniezione letale

Periodo detenzione: 22 dicembre 1978 - 10 maggio 1994

\section{Risultanze perizia psichiatrica: cenni}

In prigione Gacy tentò di invocare l'infermità mentale incolpando dei delitti il suo alter ego malvagio, tale "Jack", ma senza riuscire a convincere gli psichiatri del carcere che lo giudicaro$\underline{\text { no in grado di intendere e volere. Secondo rapporti medici }}$ pubblicati, John Wayne Gacy era uno psicopatico riconosciuto che non mostrava alcun rimorso per i crimini commessi. L'ultima dichiarazione al suo avvocato prima dell'esecuzione fu che "ucciderlo non avrebbe comunque compensato la perdita delle vite degli altri e che lo Stato lo stava assassinando". Le ultime parole del condannato prima della morte furono semplicemente: «Baciatemi il culo!» (Kiss my Ass!)

\section{Scrittura di John Wayne Gacy}

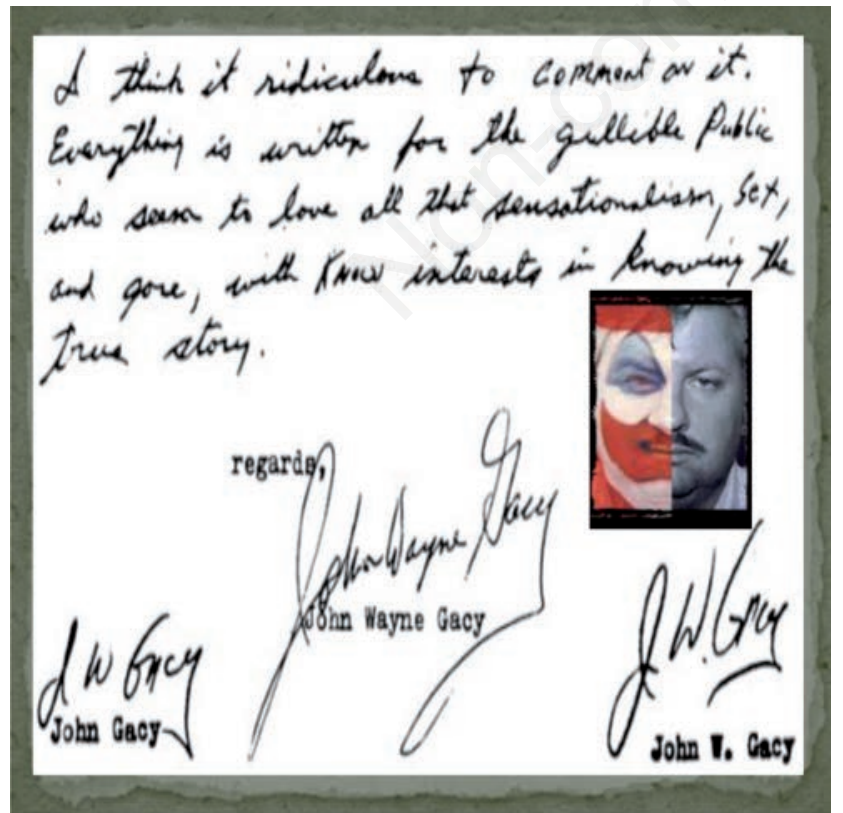

Principali caratteristiche della scrittura, mirate all'evidenziazione di tratti distintivi inerenti alle seguenti problematiche: Narcisismo, Aggressività, Impulsività, Sessualità, Relazioni interpersonali
- Spazio riempiti completamente (narcisismo)

- I margini: sinistro ben tenuto, superiore assente, destro progressivo (bisogno di sicurezza, paura dell'inconscio, imprudenza nei rapporti interpersonali);

- Direzione del rigo variabile tendenzialmente ascendente e discendete a fine rigo (ricerca di adattamento, compensa un'insicurezza di fondo. Tono dell'umore variabile);

- Legata (perseveranza nelle decisioni);

- Rapida (aumento dell'impazienza);

- Larga tra parole (guardingo nei rapporti interpersonali);

- Pressione del tratto marcata (necessità di dominio sugli altri);

- Oscura (il testo incomprensibile, indica volontà di occultamento);

- Tagli “ $t$ ” variabili (avanzati) [compensazione con eccesso di sicurezza], arretrati [paure], a laccio [aumento dell'impazien$\mathrm{za}$; (compensa le paure esistenziali con eccessiva sicurezza di se, unita alla spinta compulsiva);

- Puntini sulle "i" accentati (ansia nel collocamento delle cose $=$ insicurezza);

- Virgole grandi e distaccate dal testo (tendenza alla spinta aggressiva);

- Asole delle lettere "g" rifiutate (non reale interesse per la sessualità, ma mirata solo al predominio);

- Lettera " $y$ " nella firma - riccio del nascondimento (tendenza all'occultamento - pensieri e azioni);

- Non identità tra testo e firma (il vissuto del proprio 'sé' e l'etichetta sociale esibita, sono rappresentati in modo distinto);

- Firma con iniziali grandi, il gesto grafico si schiaccia nel procedere divenendo oscuro, con gesti allunghi lanciati, acuminati e regressivi (vissuto del proprio 'sé' narcisistico, insicurezza di fondo, rifiuto, forte spinta aggressiva).

\section{Profilo grafologico (sintesi)}

La scrittura di Gacy è caratterizzata da forte spinta aggressivo-compulsiva, che non riusciva a controllare con conseguente discontrollo degli impulsi. Tra le altre caratteristiche, interessante quanto emerso dalla grafia, che evidenzia 'non identità' tra testo e firma, tipico segnale di allarme in questi casi dove il tratto distintivo è quello di esibire una personalità diversa da quella reale. L'esagerata inclinazione della scrittura fino a schiacciarsi e a diventare oscura delinea un comportamento imprudente verso l'altro che non conta nulla se non come strumento per i propri fini.

Si presenta apparentemente sicuro, tratto che compensa adottando un comportamento prevaricatore ed oppositivo. Le relazioni in particolar modo quelle intime, sono rifiutate e strumentalizzate.

La Firma molto lanciata esageratamente grande e sottolineata traccia un profilo narcisista ed aggressivo, confermato dalla punteggiatura grande e distaccata dal testo (virgole).

Evidenzia inoltre problematiche nella sessualità rifiutata $\mathrm{e}$ rivolta solo al predominio sull'altro, a causa di paure ed insicurezze di fondo che teneva ben occultate.

Come personalità, si avvicina più a Bundy e meno a Dahmer. Rispetto a quanto commesso non evidenzia alcun pentimento, scevro da qualsivoglia senso di colpa, fornendo a sé stesso una personale spiegazione, con comportamento sadico e cinico.

Appartiene alla categoria del S.K. del 'Controllo del Potere'. Ritenuto capace di intendere e di volere, quindi, 'sano di mente'. 


\section{Conclusioni}

Dallo studio effettuato e relativo alla disamina (nel presente articolo), di 3 tipi di Serial Killer appartenenti alla tipologia del "Controllo del Potere", quanto emerso dai rispettivi 'Profili grafologici', va a confermare non soltanto le ricerche della letteratura corrente sul tema, ma anche quanto emerso dalle risultanze delle Perizie Psichiatriche espletate da parte di autorevoli esperti.

Dahmer omosessuale, personalità timida ed inadeguata con evidenti problematiche affettivo-emotive e sessuali, che ha rivolto la propria aggressività repressa verso soggetti maschi e di colore. Bundy deluso anche lui a livello affettivo, ha rivolto la sua prorompente ed aggressiva personalità, verso quelle donne che in qualche modo evidentemente, lo riportavano a vissuti di un passato rimosso ma mai dimenticato. Gacy, pedofilo con forti problematiche nella sessualità con probabile omosessualità latente e che secondo quanto recita la sua storia biografica, sembrerebbe emergere una relazione disfunzionale con la figura paterna che lo ha maltrattato e ridicolizzato durante l'infanzia. All'età di nove anni, viene abusato sessualmente da un amico di famiglia.

Sia pur per fattori diversi, prevalentemente mossi dalle stesse motivazioni: delusione, rabbia, aggressività, ferita narcisistica insanabile. Tali vissuti, li hanno rivolti nell'unico modo per loro possibile, quello di rifarsi solo apparentemente del dolore subito in passato, insopportabile per delle personalità come le loro. Nella realtà così come nella fiction, i serial killer vengono definiti mostri, psicopatici alla continua ricerca di prede mirate, per soddisfare il loro piacere impulsivo e 'malato'. Caratterizzati psicodinamicamente da specifiche componenti quali ad esempio:

- Timore di perdere la stima di sé

- Profonde aspirazioni narcisistiche

F- rustrazioni subite in tal senso

- Estrinsecazione della volontà di potenza compensatoria (il cosiddetto 'sé grandioso patologico' di Klein e Mahler o formazione reattiva di superiorità nei confronti dei propri profondi sentimenti di inferiorità

- Il 'Narcisismo maligno di Kernberg'

- Abnormi timori abbandonici a causa spesso dell 'inesistente del tutto, strutturazione del fisiologico complesso edipico e.......

(Palermo - Mastronardi Il Profilo Criminologico pag. 153).

Correspondence: Monica Calderaro.

E-mail: calderaromonica6@gmail.com

Key words: graphology, criminology, serial killer, psychographology, aggression, sexuality.

Parole chiave: grafologia, criminologia, serial killer, psicografologia, aggressività, sessualità.

Palabras clave: grafología, criminología, asesino en serie, psicografía, agresión, sexualidad.

Received for publication: 2 September 2020.

Accepted for publication: 9 November 2020.

This article is distributed under the terms of the Creative Commons Attribution Noncommercial License (by-nc 4.0) which permits any noncommercial use, distribution, and reproduction in any medium, provided the original author(s) and source are credited.

${ }^{\circ}$ Copyright: the Author(s), 2020

Licensee PAGEPress, Italy

Rivista di Psicopatologia Forense, Medicina Legale, Criminologia 2020; $25: 321$

doi:10.4081/psyco.2020.321
La Grafologia sta acquistando sempre più attenzione da parte della Comunità scientifica, che in diversi casi, come ad esempio quello applicato nel contesto criminologico-forense, propone un utilizzo sempre più innovativo del Test grafologico, che va a completare una panoramica onnicomprensiva fornita dalla letteratura corrente, nonché da studi e ricerche sempre più disponibili, ed in alcuni casi a compensare eventuali lacune .

Per questo l'auspicio di chi scrive, è che ogni operatore del settore, sia quello di compiere gli sforzi necessari per fare in modo che questa Disciplina, acquisti sempre più spazi operativi, (evitando che alcuni la utilizzino come test da salotto), dandogli la giusta connotazione scientifica, a tutto vantaggio di coloro che operano in questo ambito.

\section{Bibliografia}

Angioni M., Fratoni F., Straccamore I., "Appunti di criminologia e criminalistica. Note ed approfondimenti su vecchie tecniche e nuove scienze dell'investigazione", Libreria Universitaria Benedetti, L'Aquila, 2010.

Calderaro M., "Slides lezioni master di criminologia" e "Corso di grafologia" Sapienza Università di Roma.

Douglas J.E., Burgess A.W., Burgess A.G., Ressler R.K., "Crime classification manual - il manuale dell'FBI sulla classificazione e investigazione dei crimini violenti" (Prima edizione italiana a cura di Massimo Picozzi), CSE Ed., Roma, 2008.

Mastronardi V., Bidoli S., Calderaro M., "Grafologia giudiziaria e psicopatologia forense (metodologia di indagine nel falso grafico e la capacità di intendere e di volere dalla grafia giurisprudenza)", Giuffrè Ed., Milano, 2010.

Mastronardi V. "Manuale per operatori criminologici e psicopatologi forensi" (V ediz.), Giuffrè Ed., Milano 2012.

Mastronardi V., De Luca R., "I serial killer", Newton \& Compton, 2013.

Newton M., "Dizionario sui serial killer", Grandi Manuali Newton, Newton \& Compton Ed., Roma, 2004.

Palermo G.B., Mastronardi V., "Il profilo criminologico. Dalla scena del crimine ai profili sociopsicologici”, Giuffre' Ed. 2010 .

\section{Sitografia}

http://www.lextra.info/george-b-palermo-tarquiniese-milwaukee/ Pubblicato il 19 aprile 2014,

\section{Filmografia}

Dahmer - Il cannibale di Milwaukee (2002) scritto e diretto da David Jacobson e interpretato da Jeremy Renner.

Ted Bundy (2002) scritto e diretto da Matthew Bright e interpretato da Michael Reilly Burke

Ted Bundy - Serial Killer, (2003) diretto da Paul Shapiro e interpretato da Billy Campbel.

Il mostro (1986) (sceneggiato tv), diretto da Marvin J. Chomsky e interpretato da Mark Harmon

Gacy (2003) film diretto da Clive Saunders e interpretato da Mark Holton: John Wayne Gacy (adulto); Scott Alan Henr: John Wayne Gacy (ragazzo); Adam Baldwin: John Gacy Sr. 